Delft University of Technology

\title{
Radio-Frequency Reflectometry in Silicon-Based Quantum Dots
}

Liu, Y. Y.; Philips, S. G.J.; Orona, L. A.; Samkharadze, N.; McJunkin, T.; Macquarrie, E. R.; Eriksson, M. A.; Vandersypen, L. M.K.; Yacoby, A.

DOI

10.1103/PhysRevApplied.16.014057

Publication date

2021

Document Version

Final published version

Published in

Physical Review Applied

\section{Citation (APA)}

Liu, Y. Y., Philips, S. G. J., Orona, L. A., Samkharadze, N., McJunkin, T., Macquarrie, E. R., Eriksson, M. A., Vandersypen, L. M. K., \& Yacoby, A. (2021). Radio-Frequency Reflectometry in Silicon-Based Quantum Dots. Physical Review Applied, 16(1), [014057]. https://doi.org/10.1103/PhysRevApplied.16.014057

\section{Important note}

To cite this publication, please use the final published version (if applicable).

Please check the document version above.

\section{Copyright}

Other than for strictly personal use, it is not permitted to download, forward or distribute the text or part of it, without the consent of the author(s) and/or copyright holder(s), unless the work is under an open content license such as Creative Commons.

Takedown policy

Please contact us and provide details if you believe this document breaches copyrights.

We will remove access to the work immediately and investigate your claim. 


\title{
Radio-Frequency Reflectometry in Silicon-Based Quantum Dots
}

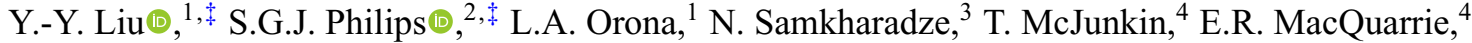 \\ M.A. Eriksson, ${ }^{4}$ L.M.K. Vandersypen $\odot,{ }^{2, *}$ and A. Yacoby ${ }^{1, \dagger}$ \\ ${ }^{1}$ Department of Physics, Harvard University, Cambridge, Massachusetts 02138, USA \\ ${ }^{2}$ QuTech and the Kavli Institute of Nanoscience, Delft University of Technology, Delft, 2600 GA, Netherlands \\ ${ }^{3}$ QuTech and Netherlands Organization for Applied Scientific Research (TNO), Delft 2628 CJ, Netherlands \\ ${ }^{4}$ University of Wisconsin-Madison, Madison, Wisconsin 53706, USA
}

(Received 31 December 2020; revised 22 May 2021; accepted 24 June 2021; published 23 July 2021)

\begin{abstract}
Radio-frequency (rf) reflectometry offers a fast and sensitive method for charge sensing and spin readout in gated quantum dots. We focus in this work on the implementation of rf readout in accumulation-mode gate-defined quantum dots, where the large parasitic capacitance poses a challenge. We describe and test two methods for mitigating the effect of the parasitic capacitance, one by on-chip modifications and a second by off-chip changes. We demonstrate that on-chip modifications enable high-performance charge readout in $\mathrm{Si} / \mathrm{Si}_{x} \mathrm{Ge}_{1-x}$ quantum dots, achieving a fidelity of $99.9 \%$ for a measurement time of $1 \mu \mathrm{s}$.
\end{abstract}

DOI: 10.1103/PhysRevApplied.16.014057

\section{INTRODUCTION}

Quantum computing promises a significant speedup of computational tasks that are practically impossible to solve on conventional computers [1-4]. Of the physical platforms available, spin-based quantum bits (qubits) in semiconductors are particularly promising [5,6]. Single-qubit gates with fidelities above $99.9 \%$ [7] and two-qubit gate fidelities up to $98 \%$ [8,9] have been demonstrated. Spin qubits in silicon are considered a strong candidate for realizing a large-scale quantum processor due to the small qubit dimensions, the localized nature of the control, the CMOS compatibility, the long coherence times [10], and the possibility of operating beyond $1 \mathrm{~K}[11,12]$.

Charge sensing is an important technique for measuring spin qubits, as their long-lived spin states can be converted into detectable charge states $[13,14]$. To detect a charge state, a sensing dot (SD) is placed in close proximity ( $d<\sim 300 \mathrm{~nm}$ ) to the qubit, as shown in Fig. 1(a). The resistance of the SD is strongly dependent on the charge state. However, measuring this resistance in dc with an amplifier at room temperature requires an integration time on the order of $30 \mu \mathrm{s}-1 \mathrm{~ms}$, due to the presence of noise and the $R C$ time constant from the line capacitance and the amplifier input impedance [11,13]. This slow readout forms a bottleneck when performing spinqubit experiments, since initialization and manipulation

\footnotetext{
*1.m.k.vandersypen@tudelft.nl

†yacoby@g.harvard.edu

$\$$ These authors contributed equally to this work.
}

can be performed on the nanosecond or microsecond scale [15-17].

Radio-frequency (rf) reflectometry [18] has been successfully applied to depletion-mode GaAs quantum dots and has enabled single-shot readout with only a few microseconds of integration time [19]. However, in accumulation-mode devices, the large parasitic capacitance of the accumulation gates to the two-dimensional electron gas (2DEG) below provides a low-impedance leakage pathway to ground for the rf signal, complicating rf-reflectometry measurements. Previous works have addressed this problem by the use of circuit-board elements [20] and careful gate design [21,22].

In this work, we further develop the theoretical model of the leakage pathway introduced by this parasitic capacitance and apply it to two methods of circumventing the impact of the parasitic capacitance. We first apply this model in the "Ohmic-style" implementation, similar to GaAs, where the signal is sent through an Ohmic contact. For this approach, we mitigate the effects of the capacitance by optimizing the on-board elements and the device design. Second, we present the "split-gate style," where the rf signal is carried by a gate that is capacitively coupled to the 2DEG [20]. By an adaptation of the sample design, the leakage pathway to the Ohmic contact is blocked by a highly resistive channel.

\section{RADIO-FREQUENCY REFLECTOMETRY}

When performing rf readout, a fixed frequency signal is applied to a SD [Fig. 1(c)]. The reflectance of the applied 


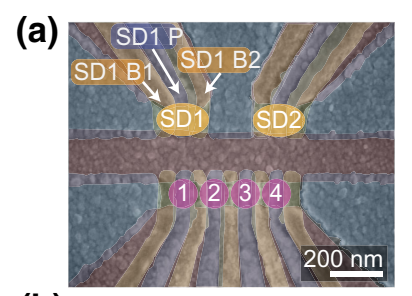

(b)

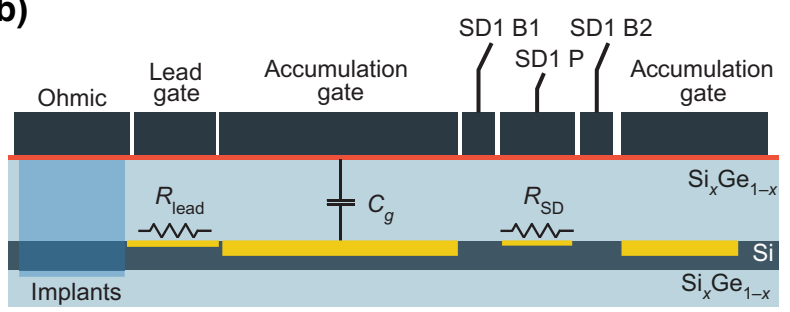

(c)

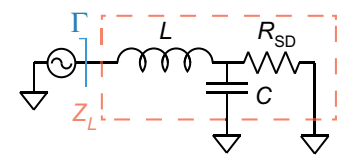

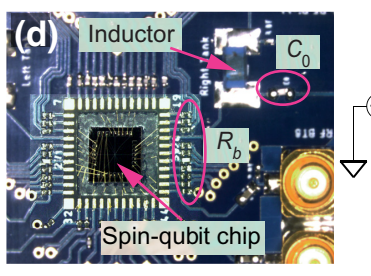
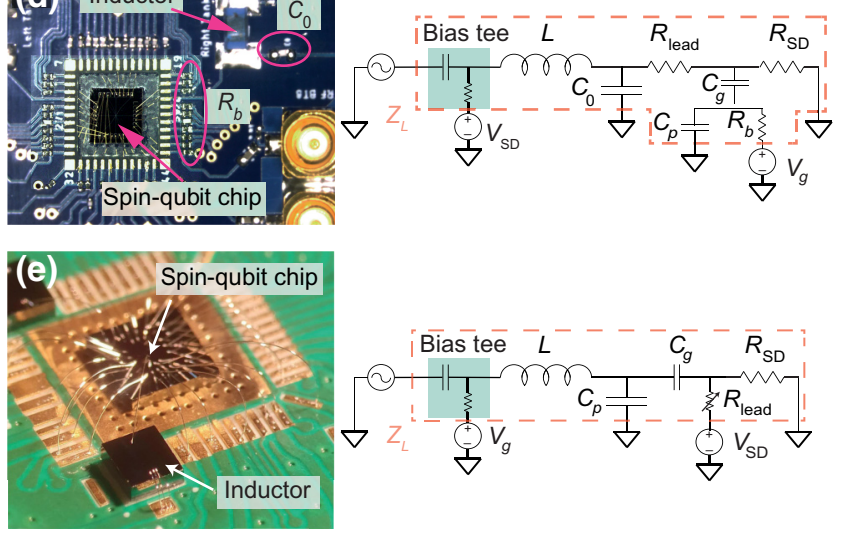

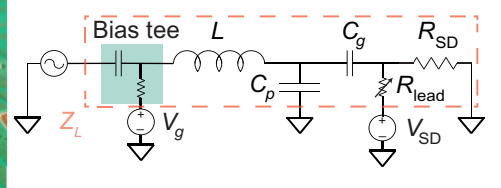

FIG. 1. (a) A false-colored SEM image of a $\mathrm{Si} / \mathrm{Si}_{x} \mathrm{Ge}_{1-x}$ device, similar to the one used in this work. (b) A schematic cross section of the sample, showing the $\mathrm{Si} / \mathrm{Si}_{x} \mathrm{Ge}_{1-x}$ quantum well and the gate stack on top. The yellow regions indicate a finite electron density in the quantum well. The three gates SD1 are used as plunger and barrier gates of the SD, with resistance $R_{\mathrm{SD}}$. The accumulation gate induces a 2DEG connecting the quantum dot to the Ohmic contact, via a smaller area controlled by the lead gate. We use the lead gate to set the resistance $R_{\text {lead }}$. The path between the bond wire to the Ohmic contact and the quantum dot also contains a contact resistance and the resistance of the 2DEG below the accumulation gate, both of which we absorb into $R_{\text {lead }}$, for simplicity. The capacitance between the $2 \mathrm{DEG}$ and the gates is dominated by $C_{g}$. (c) The circuit diagram, showing an $L C R$ circuit containing $R_{\mathrm{SD}}$. (d) An optical image (left) and the circuit diagram (right) of the wire-bonded sample on the printed circuit board (PCB) used for the Ohmic approach. The rf signal is applied to the Ohmic contact. $C_{0}$ is a capacitor on the PCB. The $R_{b}$ are resistors on the PCB to prevent leakage of the rf signal into the dc lines (e.g., the accumulation-gate electrode). The bias tee $(R=5 \mathrm{k} \Omega, C=100 \mathrm{nF})$ is implemented on the PCB to combine $\mathrm{dc}\left(V_{\mathrm{SD}}\right)$ and rf signals. $C_{p}$ is the parasitic capacitance of the bond wire and accumulation gate to the ground plane of the PCB. (e) An optical image (left) and the circuit diagram (right) of a wire-bonded sample and inductors on the PCB used for the split-gate approach. The rf signal is applied to the accumulation gate.

signal is measured. It can be expressed as

$$
\Gamma=\left(Z_{L}-Z_{0}\right) /\left(Z_{L}+Z_{0}\right)
$$

where here $Z_{L}$ represents the load impedance of the entire circuit (including matching networks and bias tees, as applicable) and $Z_{0}$ is the input impedance, equal to the output impedance of the $\mathrm{rf}$ source $\left(Z_{0}=50 \Omega\right)$. A maximal power transfer occurs when $\Gamma=0$, which is called the matching condition $\left(Z_{0} \approx Z_{L}\right)$. Given the resistive load from the $\mathrm{SD}, R_{\mathrm{SD}}$, we can reach a matching condition by adding a matching network consisting of an inductor and a capacitor, as in Fig. 1(c) [19]. The impedance of this $L C R$ circuit is given by

$$
Z_{L}=i 2 \pi f L+\frac{1}{\left(1 / R_{\mathrm{SD}}\right)+i 2 \pi f C}
$$

For this simple $L C R$ network, matching occurs when $f_{\text {res }}=$ $1 /\left(2 \pi \sqrt{L C_{0}}\right)$ and $R_{\mathrm{SD}}=L / C_{0} Z_{0}$. In general, we denote the frequency and SD resistance for the matching condition as $f_{M}$ and $R_{M}$ throughout. Ideally, matching occurs where $R_{M}$ is reached at the flank of the SD Coulombblockade peak, where the SD resistance is most sensitive to the charge occupation of the qubit dot, typically in the range of $50-500 \mathrm{k} \Omega$.
In $\mathrm{Si} / \mathrm{Si}_{x} \mathrm{Ge}_{1-x}$ quantum dots, the large parasitic capacitance from the $2 \mathrm{DEG}$ to the accumulation gate $\left[C_{g}\right.$ in Fig. 1(b)] makes it hard to find a practical matching condition. The large $C_{g}$ (approximately $1 \mathrm{pF}$ ) can be compensated by increasing the inductance of the inductor but this causes the resonance frequency to drop to a regime where most cryogenic amplifiers do not perform well (below 50-100 MHz). We solve these problems by slightly altering the circuit. We consider two approaches, which we explain in more detail below:

(a) The Ohmic approach - the rf signal is sent through the Ohmic contact. The effect of $C_{g}$ is mitigated by optimizing the circuit board and sample design [Fig. 1(d)].

(b) The split-gate approach - the accumulation gate is split into two parts. The rf signal is carried to the SD using the large $C_{g}$ between the accumulation gate and the 2DEG below [Figs. 1(b) and 1(e)]. The lead gate $\left(R_{\text {lead }}\right)$ is used to create a high-impedance path to the Ohmic.

These two approaches are tested on quadruple quantum dot devices on a $\mathrm{Si} / \mathrm{Si}_{x} \mathrm{Ge}_{1-x}$ heterostructure. Figure 1(a) shows a SEM image of a typical device. Four quantum dots are formed with the lower set of gates of the device and two sensors are formed with the upper set of gates. Large accumulation gates control the electron density from 
the quantum dots to the Ohmic contacts, approximately 50 $\mu \mathrm{m}$ away.

\section{OHMIC APPROACH}

The Ohmic approach is shown in Fig. 1(d) and introduces the rf signal to the lead of the SD through the Ohmic contact. The large $C_{g}$ and $R_{\text {lead }}$ prohibit application of the simple $R L C$ model to accumulation-mode $\mathrm{Si}_{x} \mathrm{Ge}_{1-x}$ devices. The gates are connected to ground by two channels: the line resistance $R_{b}$ to the input lines for the gate voltages $V_{g}$, which serves as rf ground, and the parasitic capacitance $C_{p}$ to ground from all the metal on the sample side of $R_{b}$ (gate, bond wire, bond pad, PCB trace). We will begin by exploring how the tank-circuit parameters $\left(R_{b}, C_{p}, C_{0}\right.$, and $\left.L\right)$ and the device parameters $\left(R_{\text {lead }}\right.$ and $C_{g}$ ) affect the matching conditions $\left(f_{M}\right.$ and $\left.R_{M}\right)$. This understanding will then be applied to demonstrate several key strategies that allow for Ohmic-style rf reflectometry in $\mathrm{Si} / \mathrm{Si}_{x} \mathrm{Ge}_{1-x}$ accumulation-mode devices. The goal is to achieve $R_{M}$ and $f_{M}$ values that are experimentally achievable and to ensure that the majority of the power is dissipated in $R_{\mathrm{SD}}$, resulting in a usable signal-to-noise ratio (SNR). For that, we aim at a $R_{M} \sim 50-500 \mathrm{k} \Omega$ and $f_{M} \sim 100-300 \mathrm{MHz}$

\section{A. Prevent shunting to ground through $C_{g}$}

The rf signal in the lead 2DEG has a low impedance path to the accumulation gates through $C_{g}$ [20]. In order to block this pathway, we design the printed circuit board (PCB) to have surface-mount resistors to increase $R_{b}$ between the sample bond pads and input lines for gate voltages $V_{g} . C_{p}$ is in parallel to $R_{b}$ and limits the ability to decrease the impact of $C_{g}$ by just increasing $R_{b}$. We place the blocking resistors close to the bond pads to minimize the amount of metal on the sample side and thereby reduce $C_{p}$. In the end, we find a minimum $C_{p}=0.2 \mathrm{pF}$. At $f>10 \mathrm{MHz}$, this would be a leakage path with a resistance smaller than $80 \mathrm{k} \Omega$. The role of $C_{g}, R_{b}$, and $C_{p}$ together can be represented by one effective gate capacitor $C_{g}^{*}=C_{p} C_{g} /\left(C_{p}+C_{g}\right)=0.2 \mathrm{pF}$ for any $R_{b}>100 \mathrm{k} \Omega$ at our target frequency range, because $C_{p}$ is the more dominant leak channel compared to $R_{b}$.

\section{B. Solution of lumped-element model}

The simple $L C R$ model always has a physically meaningful solution of $f_{M}$ and $R_{M}$ for the impedance-matching condition. However, device simulations and experiments demonstrate that large values of $R_{\text {lead }}$ and $C_{g}^{*}$ can prevent there being a $R_{M}$ and $f_{M}$ and therefore the ability to use the tank circuit for charge detection. In Fig. 2, we explore the dependence of the matching conditions on $C_{0}, L, C_{g}^{*}$, and $R_{\text {lead }}$. Simulations are performed by solving for $R_{\mathrm{SD}}$ and $f$ such that the circuit impedance $Z$ matches $Z_{0}=50 \Omega$,
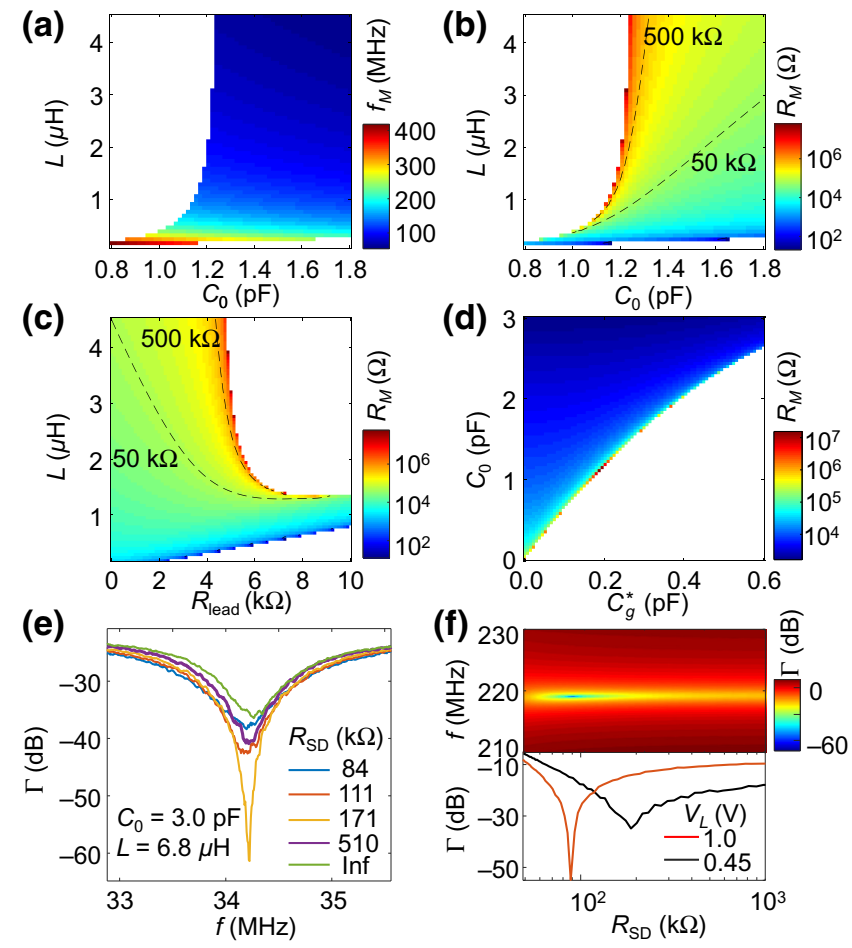

FIG. 2. (a),(b) Simulations of $f_{M}$ and $R_{M}$ as a function of $C_{0}$ and $L$ with fixed parameters of $C_{g}^{*}=0.2 \mathrm{pF}$ and $R_{\text {lead }}=3 \mathrm{k} \Omega$. The white regions are where no matching can be achieved. (c) A simulation of $R_{M}$ as a function of $R_{\text {lead }}$ and $L$ with fixed $C_{g}^{*}=0.2$ $\mathrm{pF}$, and $C_{0}=1.6 \mathrm{pF}$. (d) A simulation of $R_{M}$ as a function of $C_{0}$ and $C_{g}^{*}$ with $L=1 \mu \mathrm{H}$ and $R_{\text {lead }}=3 \mathrm{k} \Omega$. (e) An experimental demonstration of best matching with $f_{M}=34 \mathrm{MHz}$ and $R_{M}=$ $170 \mathrm{k} \Omega$. (f) The upper panel shows $\Gamma$ measured as a function of $R_{\mathrm{SD}}$ and $f$ for an optimized device and circuit board, while the lower panel shows $\Gamma$ at $f_{M}$ (red and black) as a function of $R_{\mathrm{SD}}$ when $V_{L}=1 \mathrm{~V}, 0.45 \mathrm{~V}$, respectively.

giving $R_{M}$ and $f_{M}$, respectively. The constraints that $f_{M}$ is real and that $R_{M}$ is real and positive result in there being conditions where no matching can be achieved, which are shown as white regions in Figs. 2(a)-2(d).

\section{Control matching with $C_{0}$ and $L$}

When a sample is fabricated, $C_{g}$ and $R_{\text {lead }}$ are roughly fixed, meaning that the only way to change $R_{M}$ and $f_{M}$ is through the tank-circuit parameters $L$ and $C_{0}$. We present solutions of $f_{M}$ in Fig. 2(a) and $R_{M}$ in Fig. 2(b) as a function of $L$ and $C_{0}$, with $C_{g}^{*}=0.2 \mathrm{pF}$ and $R_{\text {lead }}=3 \mathrm{k} \Omega$. We note that far from the nonmatching regions, the behavior is approximately that of the standard $L C R$ model. Under these conditions, $C_{0} \gg C_{g}$, which means that $C_{0}$ dominates the capacitance of the loaded tank circuit. When $C_{0}$ is comparable to or smaller than $C_{g}^{*}, R_{M}$ diverges.

In order to tune $C_{0}$ and $L$, our PCB is designed with solder pads for a surface-mount inductor, $L$, and a surface-mount capacitor to control $C_{0}$. The board 
parasitic capacitance also provides a significant contribution (approximately $1 \mathrm{pF}$ ) to $C_{0}$ and sets a lower bound for possible values of $C_{0}$. The ground plane near the tank circuit should be minimized to reduce this board parasitic capacitance, ensuring the tunability of the tank circuit by $C_{0}$ and $L$. Following the prediction of the model, we test lumped elements with $L=6.8 \mu \mathrm{H}$ and $C_{0}=3.0 \mathrm{pF}$ for a device with an estimated $R_{\text {lead }}=4 \mathrm{k} \Omega$ and $C_{g}=0.5$ $\mathrm{pF}\left(C_{g}^{*}=0.2 \mathrm{pF}\right)$. The result in Fig. 2(e) demonstrates impedance-matching behavior with a usable $R_{M}$. However, it comes at the cost of a very low and unusable $f_{M}$. In practice, we need $C_{0}$ to be as low as allowed by $C_{g}^{*}$ to guarantee a $f_{M}$ that is above $100 \mathrm{MHz}$. For this reason, it is important to reduce $C_{g}$ and thus $C_{g}^{*}$.

\section{Balancing $\boldsymbol{C}_{\boldsymbol{g}}$ and $\boldsymbol{R}_{\text {lead }}$ in sample design}

The dependence of the matching conditions is strongly dependent on $R_{\text {lead }}$, as shown in Fig. 2(c). At $R_{\text {lead }}=0$, the model is reduced to the standard $L C R$ model with an effective $C_{0}^{*}=C_{0}+C_{g}$. The range of $L$ that can achieve matching is drastically reduced as $R_{\text {lead }}$ increases, since more rf power would be dissipated by $R_{\text {lead }}$ before $R_{\mathrm{SD}}$. Reducing $R_{\text {lead }}$ is therefore key to achieving rf reflectometry. To capture the impact of $C_{g}$, we present a simulation of the dependence of $R_{M}$ on $C_{g}^{*}$ and $C_{0}$ in Fig. 2(d). We again observe that matching is only achieved when $C_{0}$ is large enough compared to $C_{g}$.

The sample design impacts both $C_{g}$ and $R_{\text {lead }}$, both of which we want to minimize, through the length $l$ and width $w$ of the accumulation gate. The knowledge that $C_{g} \propto l w$ and $R_{\text {lead }} \propto l / w$ reveals that decreasing $l$ is ideal for both parameters, while decreasing $w$ to improve $C_{g}$ comes at the cost of increasing $R_{\text {lead }}$, and vice versa. We find that $w=$ $5 \mu \mathrm{m}$ is sufficient to achieve consistent accumulation for usable $R_{\text {lead }}$ without increasing $C_{g}$ drastically. In the future, we will place the Ohmics as close to the SD as possible to limit $l$ as in Ref. [21]. The optimized result is demonstrated in Fig. 2(f), where we plot the reflected power $\Gamma$ in the upper panel as a function of $f$ and $R_{\mathrm{SD}}$. We apply $V_{L}=1$ $\mathrm{V}$ on the lead gate to fully turn it on and thus minimize $R_{\text {lead }}$. With this, we achieve both a usable $R_{M} \sim 100 \mathrm{k} \Omega$ and $f_{M}=220 \mathrm{MHz}$.

\section{E. Tuning $\boldsymbol{R}_{\text {lead }}$}

To experimentally confirm the dependence of $R_{M}$ on $R_{\text {lead }}$, we make use of the lead gate. When $V_{L}$ is small, the lead gate is partially turned on and thus leads to a larger $R_{\text {lead. }}$. The lower panel in Fig. 2(f) shows $\Gamma$ at $f_{M}$ as a function of $R_{\mathrm{SD}}$ when $V_{L}=1 \mathrm{~V}$ and $0.45 \mathrm{~V}$. The best matching is achieved with $67 \mathrm{k} \Omega$ for the minimized $R_{\text {lead }}$ and $200 \mathrm{k} \Omega$ for a larger $R_{\text {lead }}$, which agrees with the simulation in Fig. 2(c). This tunability also allows the use of fixed $C_{0}$ and $L$ for general devices, as the matching condition of the device can be tuned in situ. This tunability, however, is not ideal, since the larger $R_{\text {lead }}$ gets, more energy is lost before the sensor dot, resulting in a reduced SNR.

\section{SPLIT-GATE APPROACH}

In this approach, the rf signal is sent to the SD via the accumulation gate instead of via the Ohmic contact [Fig. 1(b)] [20]. The capacitance $C_{g}$ between the accumulation gate and the 2DEG allows the rf signal to couple in to the 2DEG, as shown in Fig. 1(e). The lead gate is used to generate a high-impedance channel to the Ohmic contact, preventing leakage of the rf signal.

We aim for similar design specifications for this method as for the Ohmic method: a matching resistance $\left(R_{M}\right)$ ranging from $200 \mathrm{k} \Omega$ to $600 \mathrm{k} \Omega$ and a matching frequency $\left(f_{M}\right)$ larger than $100 \mathrm{MHz}$. We simulate $f_{M}$ and $R_{M}$ for different circuit configurations. We estimate $C_{g}$ to be 280 fF from the sample design and $R_{\text {lead }}=10 \mathrm{M} \Omega$. We vary the parasitic capacitance $C_{p}$ (from the bond wires and the accumulation gate to the ground plane of the $\mathrm{PCB}$ ) and the inductance $L$, as these are parameters that are controllable by the device design and inductor choice. From the simulation results in Fig. 3, we find a large parameter space that achieves the desired matching condition for practical values of $L$ up to about $5 \mu \mathrm{H}$ as long as $C_{p}<0.3 \mathrm{pF}$. In this case, the circuit reduces to the standard $L C R$ model [23], given that the reactance of $C_{g}, \chi_{g}=\left(1 / 2 \pi f C_{g}\right) \ll$ $R_{\mathrm{SD}}$ and $R_{\text {lead }} \gg R_{\mathrm{SD}}$ [see Fig. 1(e)]. We also simulate the expected measurement bandwidth at the matching condition of this circuit. We only see a weak dependence of the bandwidth on $L$ and $C_{p}$. The bandwidth of the circuit ranges from 0.5 to $1 \mathrm{MHz}$.

For the devices used to demonstrate the splitaccumulation-gate approach, we estimate by simulation the total parasitic capacitance to be around $C_{p} \sim 250 \mathrm{fF}$. The parasitic capacitance is kept low using a compact gate layout and high-kinetic-inductance resonators as inductors [24]. We aim for an inductor value of $L \sim 3.4 \mu \mathrm{H}$, which
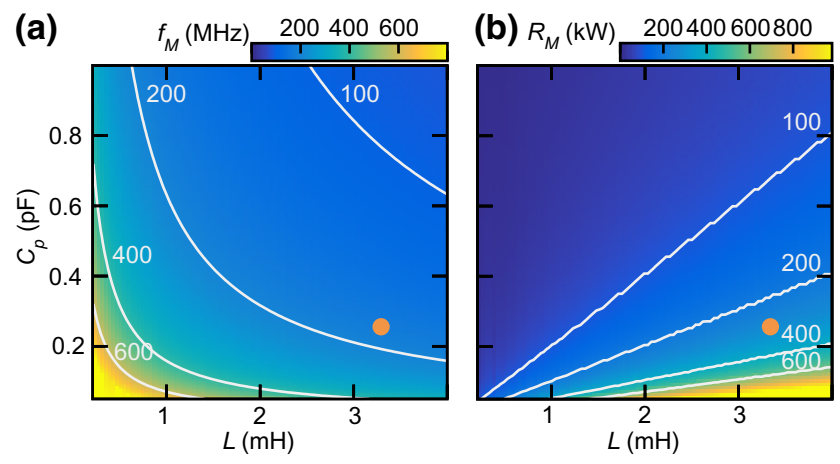

FIG. 3. Simulation results for (a) $f_{M}$ and (b) $R_{M}$ as a function of $C_{p}$ and $L$ for the split-accumulation-gate circuit when $R_{\text {lead }}=$ $10 \mathrm{M} \Omega$ and $C_{g}=280 \mathrm{fF}$. The orange dot indicates the parameters for the device and circuit used in the experiment. 
is expected to lead to a resonance frequency of approximately $180 \mathrm{MHz}$ and a matching resistance of $300 \mathrm{k} \Omega$ for the SD. When operating the device, leakage to the Ohmic contact is cut off by tuning $R_{\text {Lead }}$ above $10 \mathrm{M} \Omega$.

Figure 4(a) shows the response of the resonator versus the frequency for several values of $R_{\mathrm{SD}}$. We see that $f_{M}$ is slightly below $170 \mathrm{MHz}$. In Fig. 4(b), we find $R_{M}=$ $275 \mathrm{k} \Omega$. The circuit bandwidth can be extracted from the full width at half maximum (FWHM) of the resonance line. For $R_{\mathrm{SD}}$ equal to $R_{M}$, the bandwidth is $0.8 \mathrm{MHz}$, which means that we can measure signals up to time scales as short as approximately $600 \mathrm{~ns}$, provided that the SNR is sufficiently high. Two sensitive regions where the reflected signal depends strongly on $R_{\mathrm{SD}}$ are visible in Fig. 4(b), as indicated by the red and green shaded areas. The inset shows the expected response of the circuit in the $I-Q$ plane around $R_{M}$. The red and green regions can be differentiated by a phase $\pi$ in the measured signal. In practice, the coax line between the sample and the measurement circuit adds an unknown phase. In order to maximize the SNR, we record both $I$ and $Q$ and convert the result to a scalar.

In practice, we find that the resistance range for $R_{\mathrm{SD}}$ that gives the largest charge sensing signal is roughly $0.4-1.0 \mathrm{M} \Omega$, just above $R_{M}=275 \mathrm{k} \Omega$. This implies that we can improve the SNR by a factor of 2 by matching within this range (e.g., $600 \mathrm{k} \Omega$ ). This can be done by reducing $C_{p}$ from $250 \mathrm{fF}$ to $150 \mathrm{fF}$ (smaller gate footprint) or by increasing the inductance $L$ (see Fig. 3 ).
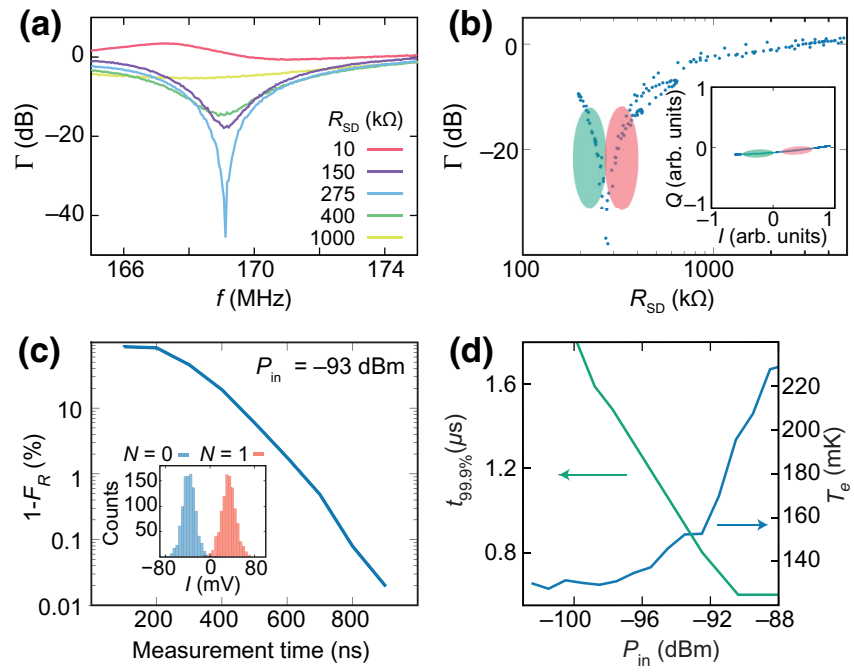

FIG. 4. The characteristics obtained with the splitaccumulation-gate approach. (a) The measured reflection coefficient as a function of $f$ for several values of $R_{\mathrm{SD}}$. (b) The reflection coefficient $\Gamma$ at $f_{M}$ as a function of $R_{\mathrm{SD}} \cdot R_{M}=275 \mathrm{k} \Omega$. The sensitive regions are marked in red and green, respectively. The inset plots the theoretical response in the $I-Q$ plane. (c) The infidelity of charge detection versus measurement time for an interdot transition. The inset shows an example histogram for calculating the fidelity. (d) $t_{99.9 \%}$ and $T_{e}$ as a function of $P_{\text {in }}$.
To characterize the readout performance experimentally, we measure the charge-readout fidelity $\left(F_{R}\right)$. This fidelity is defined as the probability of correctly determining whether a quantum dot is occupied with no $(N=0)$ or one $(N=1)$ electron. To estimate $F_{R}$, we send a train of 10000 square pulses to the target quantum dot. The dotreservoir tunnel time is several orders of magnitude shorter than the periods used in the experiment, which means that the quantum dot charge state tracks the square pulse. We sample the $I-Q$ signal for each half period of the square pulse and plot the distribution for both half periods as shown in the inset of Fig. 4(c). The overlap of the two signals is the reported infidelity $\left(1-F_{R}\right)$. For these measurements, we use a digital filter (finite impulse response type) with a pass band between $100 \mathrm{kHz}$ and $2.5 \mathrm{MHz}$. The lower frequency of the pass band is determined by the slowest signal that we want to detect $(5 \mu \mathrm{s}$ in this case). The upper frequency is taken to be larger than the bandwidth of the matching circuit, so as not to limit the measurement speed.

Figure 4(c) plots the readout infidelity $1-F_{R}$ versus the measurement time when we apply an input signal power $\left(P_{\text {in }}\right)$ of $-93 \mathrm{dBm}$ to the readout circuit (estimated from the output power at the source and the specified losses of the transmission line). We find a minimum measurement time of $t_{99.9 \%}=780 \mathrm{~ns}$ in order to achieve $F_{R}>99.9 \%$. We see that $t_{99.9 \%}$ strongly depends on the input power of the rf-readout circuit [Fig. 4(d)]. The SNR is improved by larger $P_{\text {in }}$ until the bandwidth limit of the circuit is reached $(0.8 \mathrm{MHz})$. On the other hand, larger $P_{\text {in }}$ also affects the effective electron temperature of the quantum dots. To characterize the trade-off, we measure $T_{e}$ by measuring the polarization line of an interdot transition [25] and plot the result as a function of $P_{\text {in }}$ in Fig. 4(d). We note that $T_{e}$ starts to increase dramatically once $P_{\text {in }}>-93$ $\mathrm{dBm}$. We recommend that power should only be supplied to the rf-readout circuit while the readout is being done, to prevent the readout from affecting the qubit operations.

\section{CONCLUSION}

In this work, we demonstrate two methods that can be used to achieve a reasonable matching condition for rf-reflectometry measurements in accumulation-mode devices. For the Ohmic method, we demonstrate that a series resistance can be used to reduce leakage through the parasitic capacitance. Additionally, a careful sample design is necessary in order to obtain both a workable frequency and matching resistance. With further design changes, such as moving the Ohmic contact closer to the quantum dot and drastically reducing the accumulationgate capacitance, the Ohmic method can perform as well as the split-accumulation-gate method [21,22]. For the split-accumulation-gate method, the rf source is directed to the accumulation gate of the SD and the addition of 
the lead gate allows us to efficiently cut off the leakage path to the Ohmic contact. The charge state of a qubit dot can be read out within $1 \mu$ s with a $>99.9 \%$ fidelity, which matches the state-of-the-art readout performance. The split-accumulation-gate method is useful when it is difficult to achieve a very low $C_{g}$ and/or to keep $R_{2 \mathrm{DEG}}$ sufficiently low.

\section{ACKNOWLEDGMENTS}

We acknowledge Lisa Edge from HRL Laboratories for the growth and distribution of the $\mathrm{Si} / \mathrm{Si}_{x} \mathrm{Ge}_{1-x}$ heterostructures that are used in this experiment. We acknowledge useful discussions with R. Schouten and the members of our research groups. The research was sponsored by the Army Research Office (ARO) and was accomplished under Grant No. W911NF-17-1-0274. The views and conclusions contained in this document are those of the authors and should not be interpreted as representing the official policies, either expressed or implied, of the ARO, or the U.S. Government. The U.S. Government is authorized to reproduce and distribute reprints for Government purposes notwithstanding any copyright notation herein. We acknowledge financial support from the Marie Skłodowska-Curie actions - Nanoscale solid-state spin systems in emerging quantum technologies - SpinNANO, Grant Agreement No. 676108. The Wisconsin Centers for Nanoscale Technology acknowledges financial support from the National Science Foundation (NSF) (Grant No. DMR-1625348) in the acquisition of the Elionix ELS G-100 electron-beam-lithography instrument used for part of this work.

[1] R. D. Somma, S. Boixo, H. Barnum, and E. Knill, Quantum Simulations of Classical Annealing Processes, Phys. Rev. Lett. 101, 130504 (2008).

[2] G. Carleo and M. Troyer, Solving the quantum many-body problem with artificial neural networks, Science 355, 602 (2017).

[3] A. Aspuru-Guzik, A. D. Dutoi, P. J. Love, and M. HeadGordon, Simulated quantum computation of molecular energies, Science 309, 1704 (2005).

[4] P. W. Shor, Polynomial-time algorithms for prime factorization and discrete logarithms on a quantum computer, SIAM Rev. 41, 303 (1999).

[5] J. J. Morton and B. W. Lovett, Hybrid solid-state qubits: The powerful role of electron spins, Annu. Rev. Condens. Matter Phys. 2, 189 (2011).

[6] L. Vandersypen, H. Bluhm, J. Clarke, A. Dzurak, R. Ishihara, A. Morello, D. Reilly, L. Schreiber, and M. Veldhorst, Interfacing spin qubits in quantum dots and donors, hot, dense, and coherent, npj Quantum Inf. 3, 1 (2017).

[7] K. Takeda, J. Yoneda, T. Otsuka, T. Nakajima, M. R. Delbecq, G. Allison, Y. Hoshi, N. Usami, K. M. Itoh, S. Oda, et al., Optimized electrical control of a $\mathrm{Si} / \mathrm{SiGe}$ spin qubit in the presence of an induced frequency shift, npj Quantum Inf. 4, 54 (2018).

[8] W. Huang, C. H. Yang, K. W. Chan, T. Tanttu, B. Hensen, R. C. C. Leon, M. A. Fogarty, J. C. C. Hwang, F. E. Hudson, K. M. Itoh, et al., Fidelity benchmarks for two-qubit gates in silicon, Nature 569, 532 (2019).

[9] X. Xue, T. Watson, J. Helsen, D. R. Ward, D. E. Savage, M. G. Lagally, S. N. Coppersmith, M. Eriksson, S. Wehner, and L. Vandersypen, Benchmarking Gate Fidelities in a $\mathrm{Si} / \mathrm{SiGe}$ Two-Qubit Device, Phys. Rev. X 9, 021011 (2019).

[10] F. A. Zwanenburg, A. S. Dzurak, A. Morello, M. Y. Simmons, L. C. Hollenberg, G. Klimeck, S. Rogge, S. N. Coppersmith, and M. A. Eriksson, Silicon quantum electronics, Rev. Mod. Phys. 85, 961 (2013).

[11] L. Petit, H. G. J. Eenink, M. Russ, W. I. L. Lawrie, N. W. Hendrickx, S. G. J. Philips, J. S. Clarke, L. M. K. Vandersypen, and M. Veldhorst, Universal quantum logic in hot silicon qubits, Nature 580, 355 (2020).

[12] C. H. Yang, R. C. C. Leon, J. C. C. Hwang, A. Saraiva, T. Tanttu, W. Huang, J. Camirand Lemyre, K. W. Chan, K. Y. Tan, F. E. Hudson, et al., Operation of a silicon quantum processor unit cell above one kelvin, Nature 580, 350 (2020).

[13] J. Elzerman, R. Hanson, L. W. Van Beveren, B. Witkamp, L. Vandersypen, and L. P. Kouwenhoven, Single-shot readout of an individual electron spin in a quantum dot, Nature 430, 431 (2004).

[14] C. Barthel, D. Reilly, C. M. Marcus, M. Hanson, and A. Gossard, Rapid Single-Shot Measurement of a SingletTriplet Qubit, Phys. Rev. Lett. 103, 160503 (2009).

[15] J. R. Petta, A. C. Johnson, J. M. Taylor, E. A. Laird, A. Yacoby, M. D. Lukin, C. M. Marcus, M. P. Hanson, and A. C. Gossard, Coherent manipulation of coupled electron spins in semiconductor quantum dots, Science 309, 2180 (2005).

[16] F. H. Koppens, C. Buizert, K.-J. Tielrooij, I. T. Vink, K. C. Nowack, T. Meunier, L. Kouwenhoven, and L. Vandersypen, Driven coherent oscillations of a single electron spin in a quantum dot, Nature 442, 766 (2006).

[17] D. Kim, D. R. Ward, C. B. Simmons, D. E. Savage, M. G. Lagally, M. Friesen, S. N. Coppersmith, and M. A. Eriksson, High-fidelity resonant gating of a silicon-based quantum dot hybrid qubit, Npj Quantum Inf. 1, 15004 (2015).

[18] R. J. Schoelkopf, P. Wahlgren, A. A. Kozhevnikov, P. Delsing, and D. E. Prober, The radio-frequency single-electron transistor (RF-SET): A fast and ultrasensitive electrometer, Science 280, 1238 (1998).

[19] D. Reilly, C. Marcus, M. Hanson, and A. Gossard, Fast single-charge sensing with a rf quantum point contact, Appl. Phys. Lett. 91, 162101 (2007).

[20] C. Volk, A. Chatterjee, F. Ansaloni, C. M. Marcus, and F. Kuemmeth, Fast charge sensing of $\mathrm{Si} / \mathrm{SiGe}$ quantum dots via a high-frequency accumulation gate, Nano Lett. 19, 5628 (2019).

[21] E. J. Connors, J. Nelson, and J. M. Nichol, Rapid High-Fidelity Spin-State Readout in $\mathrm{Si} / \mathrm{Si}$ - Ge Quantum Dots via rf Reflectometry, Phys. Rev. Appl. 13, 024019 (2020).

[22] A. Noiri, K. Takeda, J. Yoneda, T. Nakajima, T. Kodera, and S. Tarucha, Radio-frequency-detected fast charge sensing 
in undoped silicon quantum dots, Nano Lett. 20, 947 (2020).

[23] L. Taskinen, R. Starrett, T. Martin, A. Micolich, A. Hamilton, M. Simmons, D. Ritchie, and M. Pepper, Radiofrequency reflectometry on large gated two-dimensional systems, Rev. Sci. Instrum. 79, 123901 (2008).

[24] N. Samkharadze, A. Bruno, P. Scarlino, G. Zheng, D. P. DiVincenzo, L. DiCarlo, and L. M. K. Vandersypen,
High-Kinetic-Inductance Superconducting Nanowire Resonators for Circuit QED in a Magnetic Field, Phys. Rev. Appl. 5, 044004 (2016).

[25] C. Van Diepen, P. T. Eendebak, B. T. Buijtendorp, U. Mukhopadhyay, T. Fujita, C. Reichl, W. Wegscheider, and L. M. Vandersypen, Automated tuning of inter-dot tunnel coupling in double quantum dots, Appl. Phys. Lett. 113, 033101 (2018). 